Published in final edited form as:

Behav Neurosci. 2012 June ; 126(3): 381-391. doi:10.1037/a0028252.

\title{
The Role of Adult Hippocampal Neurogenesis in Reducing Interference
}

\author{
Paul Luu, \\ Department of Physiology, University of Toronto, Toronto, ON, M5T1N9, Canada \\ Orriana C. Sill, \\ Department of Psychology, Cornell University, Ithaca, NY \\ Lulu Gao, \\ Department of Physiology, University of Toronto, Toronto, ON, M5T1N9, Canada \\ Suzanna Becker, \\ Department of Psychology, Neuroscience and Behaviour, McMaster University, Hamilton, ON, \\ Canada \\ Jan Martin Wojtowicz, and \\ Department of Physiology, University of Toronto, Toronto, ON, M5T1N9, Canada \\ David M. Smith \\ Department of Psychology, Cornell University, Ithaca, NY
}

\begin{abstract}
Adult neurogenesis in the hippocampal dentate gyrus plays an important role in learning and memory. However, the precise contribution of the new neurons to hippocampal function remains controversial. Emerging evidence suggests that neurogenesis is important for pattern separation and for mitigating interference when similar items must be learned at different times. In the present study, we directly test this prediction using a recently developed olfactory memory task that has those specific features. In this task, rats learn two highly interfering lists of odor pairs, one after the other in either the same or in different contexts. Consistent with our hypothesis, focal cranial irradiation, resulting in selective reduction of neurogenesis within the dentate gyrus, significantly impaired the ability to overcome interference during learning of the second list. The ability to learn a single odor list was unimpaired. We also show that irradiation had no effect on learning in a hippocampal dependent spatial alternation task. Although both tasks involved learning interfering responses, the time course for learning the interfering items differed. Learning the interfering odor lists took place sequentially, over the course of several sessions, whereas learning the interfering spatial locations took place concurrently, within each session. Thus, the gradual addition of new neurons may have provided a pattern separation mechanism for the olfactory task but not for the maze task. These findings demonstrate a role for neurogenesis in resolving interference and they are consistent with models suggesting a critical role for neurogenesis in pattern separation.
\end{abstract}

\section{Keywords}

hippocampus; memory interference; adult neurogenesis; learning and memory; dentate gyrus

Corresponding Author: David M. Smith, Ph.D., Department of Psychology, 252 Uris Hall, Cornell University, Ithaca, NY 14853, Office: (607)227-0045, Fax: (607)255-8433,dms248@ cornell..edu.

Paul Luu and Orriana Sill contributed equally to this project. 


\section{Introduction}

Adult neurogenesis has emerged as an integral and crucial process within the hippocampus. Due to their strategic location in the dentate gyrus, new neurons are thought to play a key role in learning and memory as well as in some related hippocampal functions such as the regulation of drug addiction, emotions, and stress (Becker \& Wojtowicz, 2007; Deng, Aimone, \& Gage, 2010; Jacobs, van Praag, \& Gage, 2000; Noonan, Bulin, Fuller, \& Eisch, 2010; Schoenfeld \& Gould, 2011). Computational studies predict a role for new neurons in pattern separation and interference reduction on the basis of unique properties of these cells (Becker, 2005). One such property is the ability of new neurons to undergo a complete turnover while they grow and become transformed from one developmental stage to another during the course of several days to weeks (Deng et al., 2010). Behavioral tasks that included pattern separation have already been exploited in experiments (Clelland et al., 2009; Creer, Romberg, Saksida, van Praag, \& Bussey, 2010). These studies have shown that mice with reduced neurogenesis are impaired on such tasks. Paradoxically, Saxe et al (2007) observed improvement in a working memory task involving pattern separation. The discrepancy may be accounted for by the reliance upon different memory systems in these tasks, with the former (Clelland et al., 2009; Creer et al., 2010) being hippocampaldependent, whereas the latter task (Saxe et al., 2007) may rely more on extra-hippocampal working memory circuits in the prefrontal cortex. Nonetheless, all these tasks have in common that they require the animal to represent and separate events occurring almost simultaneously, or interleaved within a single experimental test session.

In contrast, Aimone et al (2006) and Becker and Wojtowicz (Becker, 2005; Becker \& Wojtowicz, 2007) proposed another form of pattern separation, for events separated by days, specifically related to turnover of new cells. Our model predicts that hippocampal neurogenesis should be critical when subjects must form two distinct memories for highly interfering items as long as the two learning experiences occur at different times so that distinct populations of new neurons are available for the encoding of each item. In the present study, we directly test this prediction using a recently developed task that has those specific features (Butterly, Petroccione, \& Smith, 2011). In this task, rats learn two highly interfering lists of odor pairs, one after the other in different contexts. For comparison, we also examined the role of neurogenesis in another hippocampal dependent task that we have used previously (blocked spatial alternation, Smith \& Mizumori, 2006b) and that also involved learning interfering responses. Thus, the present study examined the role of adult neurogenesis in pattern separation and memory interference within and across experimental sessions. In addition, recognizing a dynamic, reciprocal relationship between learning and neurogenesis, we deployed a battery of tests to estimate the number of young neurons and their rate of proliferation and survival in relation to behavioral performance.

\section{Materials and Methods}

\section{Animals and Time Line of the Study}

Fifty four male Long-Evans rats were obtained from Charles River (Quebec) in 5 batches, at approximately every two months. The animals were three months old on arrival and were kept in the animal facility at Guelph University (Ontario Canada) one week prior to any procedures. Rats in the irradiated group were anesthetized and underwent procedures for cranial irradiation at the Guelph Veterinary Clinic adjacent to the animal facility, as described previously (Winocur, Wojtowicz, Sekeres, Snyder, \& Wang, 2006). Control rats were anesthetized but were not exposed to irradiation.

Ten rats ( 6 controls and 4 irradiated) served as untrained cage controls, and were kept in small animal cages at the University of Toronto for the duration of the experiment. They 
received injections of BrdU at 4 weeks prior to the perfusion in synchrony with the trained rats. The remaining 44 irradiated and non-irradiated animals were transported by air to Cornell University (Ithaca, NY, USA) for all behavioral tests and kept there until the experiments were completed. Behavioral training began 5 weeks after the irradiation. The rats $(n=44)$ were first trained on the olfactory discrimination task, which lasted approximately 3 weeks. After completing the olfactory discrimination task, 10 of the rats (5 controls, 5 irradiated) were given injections of bromodeoxyuridine (BrdU) at $200 \mathrm{mg} / \mathrm{kg}$ (i.p.) and perfused 1 week later in order to determine whether olfactory training affects the subsequent production of new neurons.

One week after completing the olfactory task, 32 of the rats ( 8 rats in each of 4 groups, described below) began training on the plus maze task, which took up to 15 days to complete. One week prior to the plus maze task, the rats were injected with a single dose of bromodeoxyuridine (BrdU) at $200 \mathrm{mg} / \mathrm{kg}$ (i.p.) in order to determine whether plus maze training affects the survival of new neurons. The timing of the BrdU injection was planned specifically to detect changes in neuronal survival of 1-2 week old neurons during the plus maze task as described previously for other hippocampal dependent tasks (Sisti, Glass, \& Shors, 2007; Tronel et al., 2010). Because the olfactory discrimination task occurred prior to the BrdU injection we did not expect the neuronal survival of the BrdU-positive cells to be affected by the olfactory training. The animal's health was monitored throughout the duration of the study. The weights of the control and irradiated rats were monitored and did not differ (control $=544.77 \pm 11.76$, mean \pm SEM; irradiated $=550.3 \pm 13.6$ at the outset of training, $\left.\mathrm{t}_{(24)}=0.31, \mathrm{p}=.41\right)$. The animals were perfused exactly 25 days after BrdU injection, regardless of how quickly they reached the criterion in the plus maze task.

\section{Perfusion, sectioning and sampling}

Each animal was deeply anaesthetized with isofluorane and then intracardially perfused with $300 \mathrm{ml} 0.1 \mathrm{M}$ phosphate buffered saline (PBS, pH 7.4) followed by $200 \mathrm{ml}$ cold $4 \%$

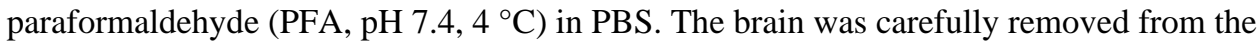
skull and placed in $4 \%$ PFA at $4{ }^{\circ} \mathrm{C}$ for 24 hours. Later, the brain was stored in $0.1 \%$ sodium azide in PBS until sectioning. The right hippocampus from each animal was carefully isolated and coronally sectioned at $30 \mu \mathrm{m}$ thickness using a vibratome. Twelve sections were sampled evenly across the whole length of the hippocampus and stained for several markers of neurogenesis as described below.

In a subset of animals ( 5 controls, 5 irradiated) the left hemisphere was sectioned coronally at $40 \mu \mathrm{m}$. Two sections from several regions of the brain were sampled representing the olfactory bulb (OB), the rostral migratory stream (RMS) and the subventricular zone (SVZ). Stereotaxic coordinates were $+6.7 \mathrm{~mm},+4.2 \mathrm{~mm},+3.2 \mathrm{~mm},+0.7 \mathrm{~mm}$ and $-0.92 \mathrm{~mm}$ (Paxinos \& Watson, 1998).

\section{CaBP/BrdU Immunohistochemistry}

Double labeling of BrdU positive cells with calcium binding protein $(\mathrm{CaBP})$ was used to identify newly-born dentate granule cells. The hippocampal sections were incubated with $1 \mathrm{~N}$ hydrochloric acid for 30 minutes at $45^{\circ} \mathrm{C}$ followed by three 5 -minute washes. The sections were incubated with anti-BrdU primary antibody (rat, 1:200 in $0.3 \%$ Triton-X PBS, Serotec) for 24 hours at $4{ }^{\circ} \mathrm{C}$ followed by three 5 -minute washes. The sections were incubated with the secondary antibody (rabbit anti-rat IgG Alexa Fluor 488, 1:200 in 0.3\% Triton-X PBS, Molecular Probes) for 2 hours at room temperature followed by three 5-min washes. Then, the sections were incubated with anti-calbindin primary antibody (rabbit, 1:200 in $0.3 \%$ Triton-X PBS, Chemicon) for 72 hours at $4{ }^{\circ} \mathrm{C}$ followed by three 5-min washes. The sections were incubated with the secondary antibody (goat anti-rabbit IgG 
Alexa Fluor 568, 1:200 in 0.3\% Triton-X PBS, Molecular Probes) for 2 hours at room temperature. Finally, the sections were washed three times and mounted on slides with mounting medium (Fluoromount, Sigma).

\section{Doublecortin Immunohistochemistry}

Doublecortin (DCX) labeling was used to identify recently born neurons. Free-floating sections were incubated with a primary goat anti-DCX antibody (1:200, Santa Cruz Biotechnology, 24 hours at $4^{\circ} \mathrm{C}$ ), followed by Alexa488 donkey anti-goat secondary antibody (1:200; Invitrogen; 2 hours at RT). All antibodies were diluted in phosphatebuffered saline containing $0.03 \%$ Triton X-100.

\section{Ki67 Immunohistochemistry}

Ki67 labeling was used to observe cell proliferation at the time of perfusion. Sections were incubated with anti-Ki67 primary antibody (rabbit, 1:200 in 0.3\% Triton-X PBS, Vector Laboratories) for 18 hours at room temperature followed by three 5-minute washes. Then, the sections were incubated with secondary antibody (goat anti-rabbit IgG Alexa Fluor 568, 1:200 in $0.3 \%$ Triton-X PBS, Molecular Probes) for 2 hours at room temperature followed by three 5-min washes. Finally, the sections were washed three times and mounted on slides with mounting medium (Fluoromount, Sigma).

\section{Cell counting}

In hippocampal sections the single-labeled cells were counted under a fluorescent microscope (40X) in the subgranular zone, excluding the upper and lower edges of the sections. The double-labeled cells ( $\mathrm{CaBP}$ and BrdU) were counted using a confocal microscope (Leica). The total number of cells (per dentate gyrus) was obtained by multiplying the average number of cells per section by the total number of sections, in each animal.

In coronal sections used for estimates of cell number in the SVZ and RMS, cells expressing BrdU were counted within the region covered by DCX-positive cells. In the OB, BrdU positive cells were counted within 4 square visual fields $(500 \times 500 \mu \mathrm{m})$ located within the area covered by NeuN-positive cells. All counts were done on a fluorescent microscope. The cells numbers are given as densities (per $\mathrm{mm}^{2}$ of area examined).

\section{General Behavioral Methods and Rationale}

We trained rats on two different behavioral tasks that we have used previously and that have been shown to be hippocampal dependent in our laboratory (Butterly et al., 2011; Smith \& Mizumori, 2006a). In experiment 1, rats learned two lists of interfering odor pairs. They learned the first list over the course of several daily training sessions, followed by training on the second list during subsequent training days. In experiment 2, the rats were trained to remember and approach two different reward locations on a plus maze. Rewards were placed on the east arm for the first half of each training session and on the west arm for the second half, so the two reward locations were learned concurrently.

\section{Experiment 1: Olfactory Discrimination Task}

Subjects were 44 adult male Long-Evans rats. Prior to training, the rats were placed on a restricted feeding regimen ( $80-85 \%$ of free feeding weight). The rats were trained to dig in cups of odorized bedding material to retrieve buried food rewards ( $45 \mathrm{mg}$ sucrose pellets, Bioserve, Inc., Frenchtown, NJ). All of the rats were first trained on one list of odor pairs. They were then given training on a second list of odor pairs either in the same context or a different context, yielding a $2 \mathrm{X} 2$ design with irradiation condition (control or irradiated) and 
context condition (same or different) as factors. One rat was excluded due to experimenter error in the training procedure, resulting in 11 rats in each group, except the control-different condition which had 10 rats.

The two contexts differed along the following dimensions: color of the chamber (white or black), color of surrounding area (either an open experimental room with black painted walls or a $6 \times 8$ feet area enclosed by white plastic blinds), substrate in the chamber (uncovered Plexiglass floor or a black rubber mat), the $65 \mathrm{~dB}$ continuous background masking noise (white noise or pink noise) and the ambient odor left by wiping out the chamber with baby wipes prior to each training session (unscented or scented, Rite Aid, Inc.).

The rats were trained in Plexiglas chambers $(45 \mathrm{~cm}$ wide $\times 60 \mathrm{~cm}$ long $\times 40 \mathrm{~cm}$ deep) equipped with a removable divider, which separated the odor presentation area from an area where the rats waited during the intertrial interval. Odor cues were presented in ceramic cups $(8.25 \mathrm{~cm}$ in diameter, $4.5 \mathrm{~cm}$ deep). The digging cups fit into circular cutouts cemented to the floor of the chamber to discourage the rats from moving the cups or tipping them over. Twenty-four pure odorants served as cues (for details see Butterly et al., 2011). Briefly, the amount of each odorant was calculated so that it produced an equivalent vapor phase partial pressure when mixed with $50 \mathrm{ml}$ of mineral oil (Cleland et al. 2002). $10 \mathrm{ml}$ of each odorant solution was then mixed with 2 liters of corncob bedding material and stored in covered containers.

Prior to training, the rats were acclimated to each of the two contexts for two ten minute sessions in order to control for possible effects of novelty on neurogenesis. The rats were then shaped to dig in cups of bedding to retrieve buried rewards. After the rats had learned to reliably retrieve the rewards from the bottom of the cups, they began training on the first of two lists of odor pairs. Each list contained 8 odor pairs (16 individual odors). The two odors comprising each pair were always presented together, in separate cups. Within each odor pair, one odor always contained a buried reward and the other did not. The predictive value of the odors (rewarded or non-rewarded) was counterbalanced across subjects and their presentation locations for each trial (left or right side of the chamber) were randomized. The daily training sessions consisted of 64 trials ( 8 trials with each odor pair, presented in an unpredictable sequence).

At the start of each trial, the experimenter placed the two cups containing the odorized bedding in the assigned locations (left or right) and removed the divider so that the rat could approach the cups. The rat was allowed to dig until he retrieved the reward. A digging response was recorded if the rat displaced any of the bedding, except when stepping into the cup without investigating. After consuming the reward, the rat was returned to the waiting area and the divider was replaced. During an inter-trial interval of approximately 10 seconds, the experimenter prepared the cups for the next trial. The rats were given daily training sessions on list 1 until they reached a behavioral criterion of $90 \%$ correct choices on two consecutive sessions.

After reaching the criterion, the rats were given 5 training sessions on a second list of 8 odor pairs. The rats were trained in either the same context where they learned the first list or in a different context. The training sessions for list 2 were carried out in the same manner as the list 1 training sessions, except that the second list contained 8 new odor pairs. In order to induce high levels of interference between the two lists, each of the new odor pairs for list 2 consisted of a novel odor and an odor which had previously been presented in list 1 . Of the 8 odors taken from list 1 , half had been rewarded previously and half had not. For example, if the first two odor pairs on list 1 were A+/B- and C+/D-, the first two odor pairs on list 2 
would be $\mathrm{X}+/ \mathrm{A}$ - and $\mathrm{D}+\mathrm{Y}$-. This ensured that the rats could not adopt a strategy of simply approaching the novel odor (or avoiding the familiar odor) within each new odor pair.

Previous studies indicated that the rats could not smell the buried rewards (Butterly et al., 2011). Nevertheless, a subset of the rats $(n=18)$ were tested to ensure that the rats were not able to directly detect the pellets. After the completion of training, the rats were given a session consisting of 24 trials ( 3 trials with each of the 8 rewarded odors from list 2). On each trial, the rats were presented with two cups containing the same odor. However, only one of the cups was baited. If the rats could directly detect the pellets, they would be expected to perform better than chance (50\%). The rats chose the baited cup $49.53 \%$ of the time, which did not differ from chance $\left(\mathrm{t}_{(17)}=.265, \mathrm{p}=.80\right)$. The long-term effects of the olfactory task on neurogenesis were measured by estimating the number of proliferating, Ki-67-positive progenitors and immature, DCX-positive neurons.

\section{Experiment 2: Plus Maze Task}

The subjects were 32 adult male Long-Evans rats which had previously been trained in the olfactory discrimination task for experiment 1 . The rats were trained to approach the east arm of a plus maze for reward during the first half of each training session and to approach the west arm during the second half. In this experiment we sought to determine whether suppression of neurogenesis would impair learning in this task and whether training on this task would increase the survival of new neurons, as has been reported in other hippocampal dependent tasks (Epp, Haack, \& Galea, 2011; Sisti et al., 2007; Tronel et al., 2010). To this end, all of the rats were given a single injection of BrdU ( $200 \mathrm{mg}$ per kg i.p.) 7 days prior to beginning training. In order to control for exercise, handling and exposure to the rewards, the rats were divided into two groups. One group received regular training sessions and the other served as a yoked control group in which each rat was given the same number of sessions as a trained rat, but they were given control sessions which did not permit learning about predictable reward locations (described below). If learning to discriminate the go east and go west trials induced neurogenesis that was greater than that seen in the yoked controls, then the neurogenesis could not be attributed to factors other than learning.

The rats were trained on a plus maze (102 $\mathrm{cm}$ across) that occupied a circular area enclosed by curtains ( $3 \mathrm{~m}$ in diameter). Distinctive objects were attached to the curtain to serve as distal visual cues. Prior to training, they were given several sessions in which they were acclimated to the plus maze and trained to retrieve chocolate milk rewards $(0.2 \mathrm{ml}$ Nestle's Quik) from cups at the ends of the maze arms. The rats in the trained condition were then given daily training trials consisting of 2 blocks of 15 trials each. During the first block of every training session, the reward was always placed at the end of the east arm. During the second block, the reward was always placed at the end of the west arm. Trials began when the rat was placed on the maze facing outward at the end of an arm and ended when the rat arrived at the reward. During an intertrial interval (ITI) of approximately 20 seconds, the rats were placed on a platform adjacent to the maze. The position of the ITI platform was constant throughout training. The start positions for each trial were randomly designated from among the 3 non-reward arms. Training continued with the same two reward locations presented each day until the rats attained a behavioral criterion of at least $75 \%$ correct choices on two consecutive sessions. Training was discontinued if the rat did not achieve this criterion in 15 days.

Each rat in the yoked control condition was given the same number of sessions as his trained counterpart. The training trials for the yoked control rats were identical to those described above, except that instead of placing the rewards in predictable locations (i.e. the east and west arms, as above), the rewards were placed on randomly designated arms. Control rats choose the rewarded arm on $64.3 \%$ of the trials, on average, over all training sessions. The 
training procedures for the yoked controls were designed to approximate this as closely as possible. On each trial, two randomly designated arms were baited (although the rat was only allowed to run until he found one of the rewards). With 2 of the 3 non-start arms baited, the rats pick a rewarded arm $66.6 \%$ of the time by chance. Additionally, analysis of the total number of arm entries confirmed that trained rats and yoked controls ran similar distances on the maze $(F[1,28]=0.71, p=.41)$. Thus, the yoked control rats were given the same number of training trials and rewards, but they could not learn to remember and approach two different predictable reward locations.

\section{Results}

\section{Effects of irradiation on neurogenesis}

Neurogenesis was selectively reduced in the dentate gyrus (DG) but not in the olfactory tract at 9 weeks and 14 weeks after irradiation (end of the study). A comparison of cell densities at 9 weeks ( $\mathrm{n}=5$ per group) and at 14 weeks ( $\mathrm{n}=16$ per group) showed a significant effect of irradiation (DCX, $F[1,41]=45.936, \mathrm{p}<0.001)$ but no main effect of time $(\mathrm{F}[1,41]=3.82$, $\mathrm{p}=0.058$ ). The average reduction of neurogenesis after 14 weeks, as measured by the number of DCX+ new neurons, was $85 \%\left(\mathrm{t}_{(30)}=9.290, \mathrm{p}<0.001\right)$. In contrast, there was no effect of irradiation on neurogenesis in the olfactory tract. The density of BrdU+ cells counted in 5 regions of the olfactory system was not affected by the irradiation procedures (ANOVA with irradiation condition and location showed no effect of irradiation, $F[1,110]=0.331, p=0.556$, and no interactions of the irradiation and location factors, Fig. 1).

\section{Experiment 1: Olfactory Discrimination Task}

Behavior-As expected, suppression of neurogenesis did not impair learning of the first list of odors. Control and irradiated rats did not differ in the number of training sessions needed to reach the criterion (irradiated mean $=4.41$ sessions, control mean $=4.14$ sessions, $\left.\mathrm{t}_{(41)}=1.06, \mathrm{p}=.30\right)$ and there were no differences between control and irradiated rats in terms of their performance on the final training session of list $1(\mathrm{~F}[1,39]=2.05, \mathrm{p}=.45)$.

Importantly, these results indicate that the irradiated rats did not have a general impairment in olfactory sensory processing or olfactory learning.

However, suppression of neurogenesis did cause a significant impairment in performance on the second list. The percent correct on each day of training were submitted to a repeated measures ANOVA with training session (5 days of training on list 2) as the within subject factor and irradiation condition (Control or Irradiated) and context condition (Same or Different) as between subjects factors (Fig. 2). This analysis revealed a significant main effect of training session $(F[1,39]=12.76, \mathrm{p}<.001)$, a significant main effect of irradiation $(F[1,39]=12.76, p<.001)$, with controls performing significantly better than irradiated subjects, and a main effect of context $(F[1,39]=4.09, p<.05)$, with subjects in the different context condition performing significantly better than subjects in the same context condition. However, there was no significant interaction of the context and irradiation conditions $(\mathrm{F}[1,39]=1.52, \mathrm{p}=.23)$. This result suggests that the suppression of neurogenesis impaired performance regardless of the context condition, in contrast to our previous findings that muscimol lesions of the dorsal hippocampus selectively impaired performance in the different context condition but not in the same context condition (Butterly et al., 2011). Whereas the muscimol lesions had no impact on performance in the same context condition, the suppression of neurogenesis may have had more widespread effect on performance, including impairment in the same context condition. This may have occurred because neurogenesis was suppressed throughout the hippocampus, whereas the muscimol lesions were specific to the dorsal hippocampus. We revisit this issue in the general discussion. 
Assessment of Interference in Each Condition-Our hypothesis was that new neurons play a beneficial role in learning because they provide a means of overcoming interference for items learned at different times. The effects of interference can be assessed by comparing performance on the two lists. When there is little opportunity for interference (e.g. when learning non-interfering material), performance on the second list is facilitated by prior experience on the first list (Butterly et al., 2011). However, if proactive interference occurs, performance on the second list will not be facilitated and can even be impaired by prior learning on the first list. Since interference is typically most pronounced during the initial stages of learning, we compared performance during the first three sessions of list 2 to performance during the same sessions of list 1 (Fig.3). A similar pattern of results was seen when all five sessions of list 2 were analyzed.

Control rats that learned the two lists in different contexts performed significantly better on the second list than on the first (paired samples $t$-test: $\left.\mathrm{t}_{(9)}=-3.96, \mathrm{p}<.005\right)$. That is, when contextual information was available to disambiguate the two conflicting lists, control rats did not experience interference and performance was facilitated on the second list. In contrast, control rats that learned the two lists in the same context showed no such facilitation (i.e. no significant change in performance from list 1 to list $2, \mathrm{t}_{(10)}=1.70, \mathrm{p}=.12$ ). Irradiated rats were not able to use contextual information to disambiguate the two lists and performance was not facilitated on list $2\left(\mathrm{t}_{(10)}=0.61, \mathrm{p}=.56\right)$. Interestingly, irradiated rats in the same context condition showed an even greater evidence of interference, as performance was significantly worse on list 2 than list $1\left(\mathrm{t}_{(10)}=2.33, \mathrm{p}<.05\right)$.

Measures of neurogenesis for the rats in the olfactory discrimination experiment were subjected to an ANOVA with irradiation condition and training condition (trained or untrained cage controls) as factors (Fig. 4). Rats in the same context and different context training groups did not differ in terms of neurogenesis (e.g. DCX, F[1,37] $=0.72, p=0.40$ ) so these groups were combined for these analyses. Although there was a main effect of irradiation (DCX labeled cells, $F[1,39]=65.22, \mathrm{p}<0.0001$ ), there was no effect of training condition (DCX, F $[1,37]=0.06, \mathrm{p}=0.82$ ) nor was there an interaction of the irradiation and training condition factors $(F[1,37]=2.846, \mathrm{p}=0.10)$. Analysis of the Ki67-positive cells revealed similar results. Thus, the olfactory learning did not have long-term effects on levels of neuronal production in terms of proliferation or neuronal differentiation.

\section{Experiment 2: Plus Maze Task}

Behavior-In contrast to the odor task, irradiated rats showed no evidence of impairment on the plus maze task. Control and irradiated rats did not differ in terms of the number of sessions needed to reach the behavioral criterion (control mean $=7.5$ sessions, irradiated mean $=9.0$ sessions, $\mathrm{t}_{(16)}=.614, \mathrm{p}=.55$, Fig. 5a). Two control rats and 3 irradiated rats failed to reach the criterion within the 15 sessions that were given. Control and irradiated rats also exhibited similar levels of performance throughout training. To assess this, the percentage of trials with a correct response were submitted to a repeated measures ANOVA with group (control and irradiated) as a between subjects factor and training stage as a within subjects factor ( 3 stages, including the first, middle and last training sessions). The rats took a variable number of training sessions to reach the criterion, so the middle training session was simply the session that was half way between the first and last session. For those rats that received an even number of sessions, the average of the two middle-most sessions was used. For example, for a rat that required 12 sessions to reach the criterion, the average performance on sessions 6 and 7 was used as the middle session. This analysis showed no difference between groups $(\mathrm{F}[1,14]=0.16, \mathrm{p}=.69)$ and no interaction of the group and training stage variables $(\mathrm{F}[1,28]=0.75, \mathrm{p}=.48$, Fig. $5 \mathrm{~b})$. The latency to reach the reward and 
various measures of inflexible behavioral responding (e.g. right or left turn biases) were also assessed and no group differences were found.

Effects of Maze Training on Neurogenesis and Survival-Neurogenesis was compared among all animals participating in the plus maze during weeks 9-14 of the study. There was a clear effect of irradiation but none of the markers (BrdU, DCX, Ki67) revealed any differences between trained rats, yoked controls, and cage controls. This lack of differences held for both non-irradiated and irradiated animals (Fig. 6).

Measures of neurogenesis (BrdU, DCX, Ki67) for the rats trained in the plus maze task were subjected to an ANOVA with irradiation condition and training condition (3 groups: trained, yoked controls and untrained caged controls) as factors. One irradiated and 3 non-irradiated animals were excluded from immunohistochemical analysis due to poor fixation of the tissue. There was a significant main effect of irradiation on the survival of BrdU+ cells born 1 week prior to the beginning of learning $(F[1,40]=40.354$, $p<0.0001)$, but there was no effect of training condition $(\mathrm{F}[1,40]=0.311, \mathrm{p}=0.51)$ nor was there an interaction of irradiation condition and training condition $(F[1,40]=0.162, p=0.162)$. Similar results were seen in the rate of maturation, as indicated by $\mathrm{CaBP} / \mathrm{BrdU}$ labeling (main effect of irradiation: $F[1,37]=26.253$, p $<0.0001$, all others n.s.) and in DCX labeled cells (main effect of irradiation: $F[1,39]=96.986, p<0.0001$, all others n.s.).

\section{Discussion}

Suppression of hippocampal neurogenesis significantly impaired performance on the olfactory discrimination task. Although performance on the first list was entirely unaffected by the loss of neurogenesis, the rats performed significantly worse than controls when they were confronted with a second list of interfering items and irradiated rats experienced significantly more interference than controls. These results therefore support accounts which suggest that hippocampal neurogenesis plays a critical role in mitigating interference (Aimone et al., 2006; Becker \& Wojtowicz, 2007).

Interestingly, irradiation produced no impairment in the plus maze task. Although the olfactory discrimination task and the maze task differ in a number of ways, both tasks are impaired by temporary inactivation of the hippocampus (Butterly et al., 2011; Smith \& Mizumori, 2006a) and both tasks induce interference. One potentially important difference between the two tasks is the different time courses for learning the interfering items. In the odor discrimination task, the rats learned the two lists of interfering items sequentially, over the course of several days. In contrast, the competing responses of the maze task were trained concurrently with both responses rewarded within each training session. Thus, the plus maze places a high demand on spatial working memory, requiring the animal to remember the current reward location and to ignore the other location. The lack of an impairment in the irradiated animals on this task is consistent with the finding that rodents with reduced neurogenesis actually outperform controls on a working memory version of the 8-arm radial maze (Saxe et al., 2007). On the other hand, the impairment seen in the sequentially learned olfactory task supports the hypothesis that the gradual addition of new neurons is an important mechanism for differentially encoding potentially interfering memories more widely separated in time (Becker \& Wojtowicz, 2007).

Specifically, we proposed the cohorts of newly-born neurons to be selectively sensitive to the incoming perforant path synaptic inputs and that they transmit the signals encoding common experiences to CA3. Following a relatively brief sensitive period of reduced firing thresholds and heightened plasticity, the cohort would progress to a further state of maturation wherein neurons are less responsive to afferent stimulation, permitting the next 
wave of young adult neurons to be preferentially recruited. The synaptic mechanisms responsible for the sensitive period include reduced GABA-ergic inhibition and enhanced NMDA-dependent plasticity (Becker \& Wojtowicz, 2007; Deng et al., 2010; Snyder, Kee, \& Wojtowicz, 2001). This putative mechanism causes similar events spaced across several days to be encoded by distinct populations of young dentate gyrus neurons. The young neurons in turn contribute to distinct memory traces being formed in downstream regions. In contrast, the maze task may not benefit from neurogenesis because concurrently learning the competing responses does not allow distinct neural populations to differentially encode them.

The odor task was specifically designed to induce interference through the use of overlapping odors on the two lists. Nevertheless, interference is also an important aspect of the maze task. It involves serial reversals of the 'go east' and 'go west' rules, which produce strong interference, and the choice point presents the rat with an array of cues that have been associated with both reward locations. This likely leads to intrusions of the memory for the incorrect reward location and most errors consisted of entries into the incorrect reward arm, rather than random entries into arms that were never rewarded (data not shown). Thus, both tasks involve interference.

Importantly, however, the type of interference differs in the two tasks. In the odor task, similar patterns (overlapping odor pairs) must be mapped to different responses. If the rat can encode the odor pairs learned in the two lists as separate events, using new neurons to generate distinctive memory traces for the overlapping inputs, the task of learning the correct response to an overlapping odor pair becomes greatly simplified as the overlap has been reduced. On the other hand, for the spatial reversal learning task, a single spatial location (the choice point) must be associated with multiple competing responses. The interference cannot be resolved by separating similar inputs, but requires learning the reward value of alternative responses to a given input. These observations suggest that the presence of interference, by itself, is not sufficient to engage neurogenesis dependent pattern separation processes. Instead, neurogenesis may be specifically beneficial for resolving the interference arising from overlapping inputs, particularly when the memories are acquired over the course of a sufficiently long timeframe.

Of course, the olfactory task and the maze task differed in other ways. For example the two tasks differ in terms of modality (visuospatial versus olfactory). However, the impairment seen in the olfactory task was not likely due to general olfactory processing deficits, since irradiation did not cause damage to the rostral migratory stream leading to the olfactory bulb, and the irradiated rats were entirely unimpaired in learning the first list of eight odor pairs. The spatial component of the maze task is probably not an important factor in the differential effects of the irradiation on the two tasks since neurogenesis has been shown to be important for some spatial tasks (Clelland et al., 2009). The observation that olfactory tasks are not consistently impaired and spatial tasks are not consistently spared, suggests that modality is not the critical factor. Moreover, the fact that the two tasks are hippocampal dependent indicates that they both engage general hippocampal mechanisms despite the modality differences.

The two tasks of the present study also differed in terms of contextual manipulations. The olfactory discrimination task involved an explicit manipulation of the environmental context whereas the maze task did not. However, our results suggested that irradiation impaired performance regardless of the contextual manipulation. This result stands in contrast to our previous finding that temporary muscimol lesions of the dorsal hippocampus selectively impaired performance in the different context condition but not in the same context condition (Butterly et al., 2011). In that study, the muscimol lesions had no discernible 
effect on performance in the same context condition, suggesting a highly specific deficit in the ability to use contextual information to resolve interference. The present results suggest that hippocampal neurogenesis may play a more general role in resolving interference regardless of whether there is an environmental contextual component.

The present results also raise the possibility that hippocampal neurogenesis may play an important role in a different kind of context. Ongoing neurogenesis may provide an internal context that gradually varies over time, allowing overlapping events to be separated into distinct memory traces when they are well separated in time, even in the absence of differentiating environmental contexts. This is consistent with the idea that ongoing neural processes form an ever changing temporal context and that individual events are embedded within this temporal context in a manner that allows for distinct representations for similar events that occur at different times (Manns, Howard, \& Eichenbaum, 2007; Polyn \& Kahana, 2008). As mentioned above, the recruitment of new neurons may serve to tag each memory trace with its own unique temporal context, thereby reducing interference (Aimone et al., 2006; Becker, 2005; Becker, Macqueen, \& Wojtowicz, 2009).

In the present study, irradiated rats exhibited proactive interference from previously learned odor pairs. Interestingly, Winocur and colleagues (2012) demonstrated retroactive interference in irradiated animals. Specifically, after animals learned distinct stimulusresponse associations based on black versus white visual cues, only those subsequently given a "confusing" interfering event, a grey cue that had no predictive value for reward, later showed impaired performance on the original black-white discrimination. Their findings were interpreted as supporting the notion that intact animals used new hippocampal neurons to form separate contextualized memories for the original and interfering events, whereas irradiated animals may have relied upon a striatal stimulus-response strategy. However, they did not exclude the possibility that the irradiated animals showed stimulus generalization after exposure to the grey cue and were no longer able to maintain the distinction between the original black and white cues. Such generalization could have accounted for their results. Our present experiments deal specifically with this possibility by showing that neurogenesis was critical for mitigating interference between overlapping associations learned at different times, and was not due to a basic deficit in olfactory stimulus discrimination.

As mentioned above, learning in the plus maze task was not impaired by irradiation. In addition, our experiments failed to show any reciprocal effect of learning on neurogenesis. There were no differences in the numbers of new neurons between rats trained in the olfactory task and untrained controls. However, since the measurements were performed at the end of the study, after the olfactory and plus maze tasks were completed, any effect of olfactory training may have been obscured by the subsequent maze training. Nevertheless, olfactory training did not produce an effect on neurogenesis that was so large that it could be detected even after maze training. However, recent studies (Dupret et al., 2007; Epp, Spritzer, \& Galea, 2007) suggest that the effects of training on neuronal survival may be subtle, with training-induced neuronal survival being restricted to specific phases of learning. One week old cells may survive at a higher rate in trained rats, while other cells born during later phases of training may show reduced survival, resulting in no net effect.

In summary, the results of this study confirm that new neurons are involved in hippocampal dependent processes that resolve memory interference. At the mechanistic level, interference between successive learning episodes may be related to the mechanism of pattern separation as proposed by theoretical models (Aimone, Wiles, \& Gage, 2009; Becker \& Wojtowicz, 2007). Further experimentation exploring these ideas seems warranted. 


\section{Acknowledgments}

This work was supported by NIH grant MH083809 to D. Smith, CIHR operating grant to J.M. Wojtowicz and NSERC discovery grant and DAS to S. Becker. Technical assistance by Ms. Yao Fang (JMW lab) is greatly appreciated. Dr. Howard Dobson and Ms. Kim Stewart provided expert help with rat irradiation procedure.

\section{References}

Aimone JB, Wiles J, Gage FH. Potential role for adult neurogenesis in the encoding of time in new memories. Nat Neurosci. 2006; 9(6):723-727. [PubMed: 16732202]

Aimone JB, Wiles J, Gage FH. Computational influence of adult neurogenesis on memory encoding. Neuron. 2009; 61(2):187-202. [PubMed: 19186162]

Becker S. A computational principle for hippocampal learning and neurogenesis. Hippocampus. 2005; 15(6):722-738. [PubMed: 15986407]

Becker S, Macqueen G, Wojtowicz JM. Computational modeling and empirical studies of hippocampal neurogenesis-dependent memory: Effects of interference, stress and depression. Brain Res. 2009; 1299:45-54. [PubMed: 19651106]

Becker S, Wojtowicz JM. A model of hippocampal neurogenesis in memory and mood disorders. Trends Cogn Sci. 2007; 11(2):70-76. [PubMed: 17174137]

Butterly DA, Petroccione MA, Smith DM. Hippocampal context processing is critical for interference free recall of odor memories in rats. Hippocampus. 2011; 21(n/a):n/a-n/a.10.1002/hipo.20953

Clelland CD, Choi M, Romberg C, Clemenson GD Jr, Fragniere A, Tyers P, Jessberger S, Saksida LM, Barker RA, Gage FH, Bussey TJ. A functional role for adult hippocampal neurogenesis in spatial pattern separation. Science. 2009; 325(5937):210-213. [PubMed: 19590004]

Creer DJ, Romberg C, Saksida LM, van Praag H, Bussey TJ. Running enhances spatial pattern separation in mice. Proc Natl Acad Sci U S A. 2010; 107(5):2367-2372. [PubMed: 20133882]

Deng W, Aimone JB, Gage FH. New neurons and new memories: how does adult hippocampal neurogenesis affect learning and memory? Nat Rev Neurosci. 2010; 11(5):339-350. [PubMed: 20354534]

Dupret D, Fabre A, Dobrossy MD, Panatier A, Rodriguez JJ, Lamarque S, Lemaire V, Oliet SH, Piazza PV, Abrous DN. Spatial learning depends on both the addition and removal of new hippocampal neurons. PLoS Biol. 2007; 5(8):e214. [PubMed: 17683201]

Epp JR, Haack AK, Galea LA. Activation and survival of immature neurons in the dentate gyrus with spatial memory is dependent on time of exposure to spatial learning and age of cells at examination. Neurobiol Learn Mem. 2011; 95(3):316-325. [PubMed: 21216298]

Epp JR, Spritzer MD, Galea LA. Hippocampus-dependent learning promotes survival of new neurons in the dentate gyrus at a specific time during cell maturation. Neuroscience. 2007; 149(2):273285. [PubMed: 17900815]

Jacobs BL, van Praag H, Gage FH. Adult brain neurogenesis and psychiatry: a novel theory of depression. Mol Psychiatry. 2000; 5(3):262-269. [PubMed: 10889528]

Manns JR, Howard MW, Eichenbaum H. Gradual changes in hippocampal activity support remembering the order of events. Neuron. 2007; 56(3):530-540. [PubMed: 17988635]

Noonan MA, Bulin SE, Fuller DC, Eisch AJ. Reduction of adult hippocampal neurogenesis confers vulnerability in an animal model of cocaine addiction. J Neurosci. 2010; 30(1):304-315. [PubMed: 20053911]

Paxinos, G.; Watson, C. The rat brain in stereotaxic coordinates. Fourth ed. New York: Academic Press; 1998.

Polyn SM, Kahana MJ. Memory search and the neural representation of context. Trends Cogn Sci. 2008; 12(1):24-30. [PubMed: 18069046]

Saxe MD, Malleret G, Vronskaya S, Mendez I, Garcia AD, Sofroniew MV, Kandel ER, Hen R. Paradoxical influence of hippocampal neurogenesis on working memory. Proc Natl Acad Sci U S A. 2007; 104(11):4642-4646. [PubMed: 17360577]

Schoenfeld TJ, Gould E. Stress, stress hormones, and adult neurogenesis. Exp Neurol. 2011 
Sisti HM, Glass AL, Shors TJ. Neurogenesis and the spacing effect: learning over time enhances memory and the survival of new neurons. Learn Mem. 2007; 14(5):368-375. [PubMed: 17522028]

Smith DM, Mizumori SJY. Hippocampal place cells, context, and episodic memory. Hippocampus. 2006a; 16(9):716-729. [PubMed: 16897724]

Smith DM, Mizumori SJY. Learning-Related Development of Context-Specific Neuronal Responses to Places and Events: The Hippocampal Role in Context Processing. Journal of Neuroscience. 2006b; 26(12):3154-3163. [PubMed: 16554466]

Snyder JS, Kee N, Wojtowicz JM. Effects of adult neurogenesis on synaptic plasticity in the rat dentate gyrus. J Neurophysiol. 2001; 85(6):2423-2431. [PubMed: 11387388]

Tronel S, Fabre A, Charrier V, Oliet SH, Gage FH, Abrous DN. Spatial learning sculpts the dendritic arbor of adult-born hippocampal neurons. Proc Natl Acad Sci U S A. 2010; 107(17):7963-7968. [PubMed: 20375283]

Winocur G, Becker S, Luu P, Rosenzweig S, Wojtowicz JM. Adult hippocampal neurogenesis and memory interference. Behavioral Brain Research. 2012; 227(2):464-469.

Winocur G, Wojtowicz JM, Sekeres M, Snyder JS, Wang S. Inhibition of neurogenesis interferes with hippocampus-dependent memory function. Hippocampus. 2006; 16(3):296-304. [PubMed: $16411241]$ 

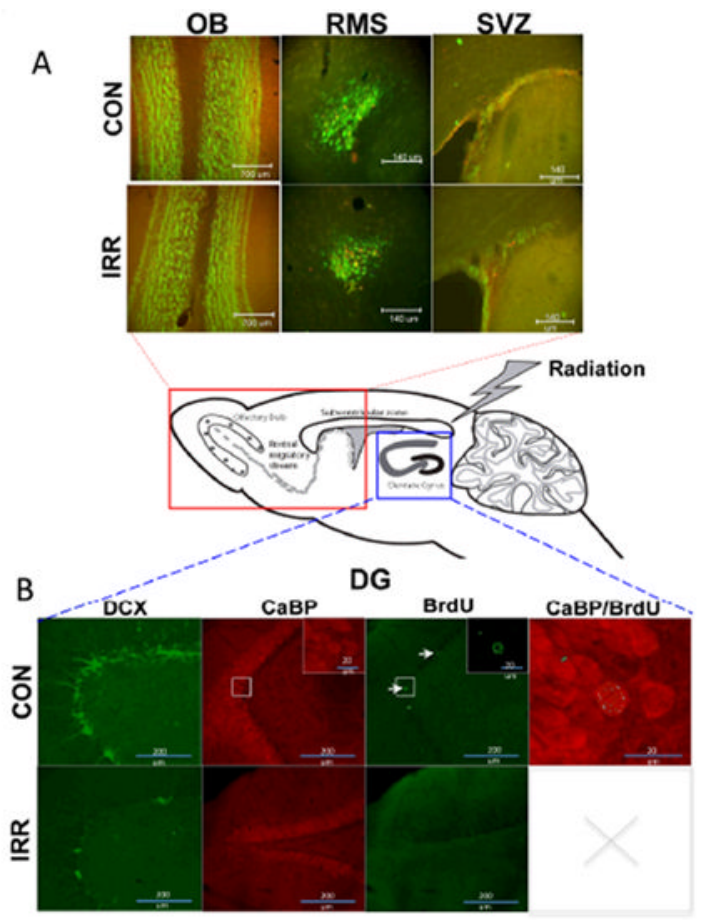

C
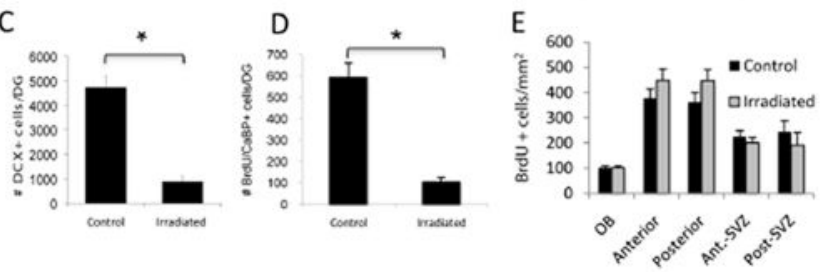

Figure 1.

Two neurogenic regions in the rat brain. Images in A show staining for NeuN (green) and BrdU (red) in the olfactory bulb (OB), and DCX (green) and BrdU (red) in the rostral migratory stream (RMS) and the subventricular zone (SVZ). Images in B show staining for DCX, CaBP, and BrdU as indicated in the dentate gyrus (DG). Irradiation was applied selectively to the rear of the brain in order to reduce neurogenesis in the DG but not in the olfactory system. There was too little $\mathrm{CaBP} / \mathrm{BrdU}$ to image in irradiated rats. Irradiation caused an $85 \%$ reduction in neurogenesis in the dentate gyrus (plot C, D), but had no effect on any of the olfactory regions (plot E). 
A.

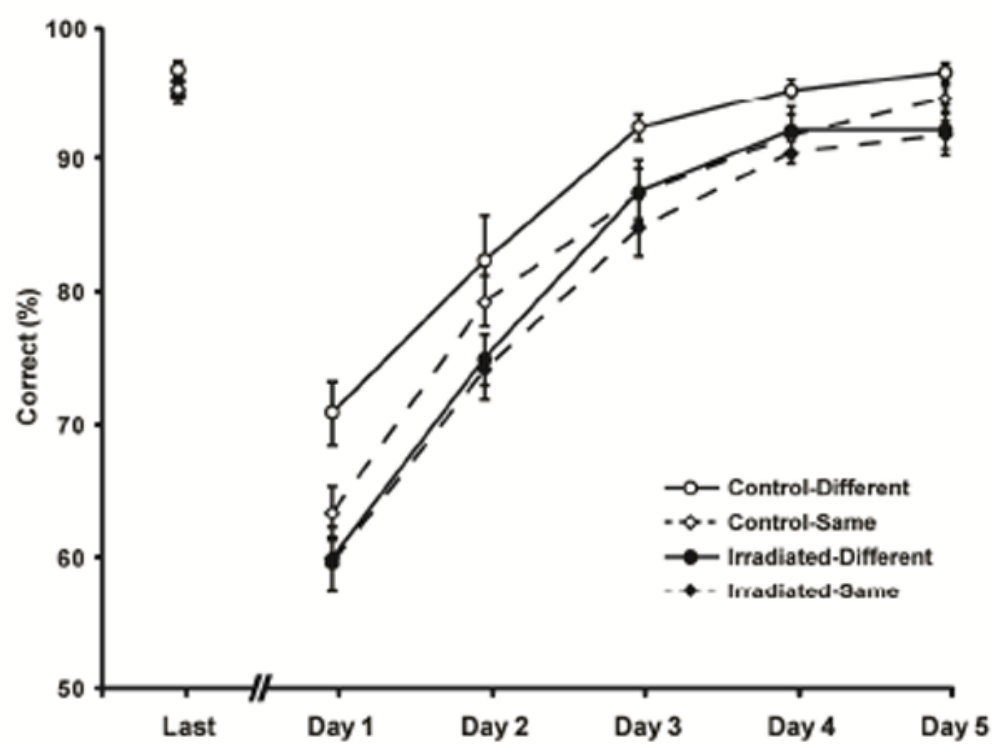

B.

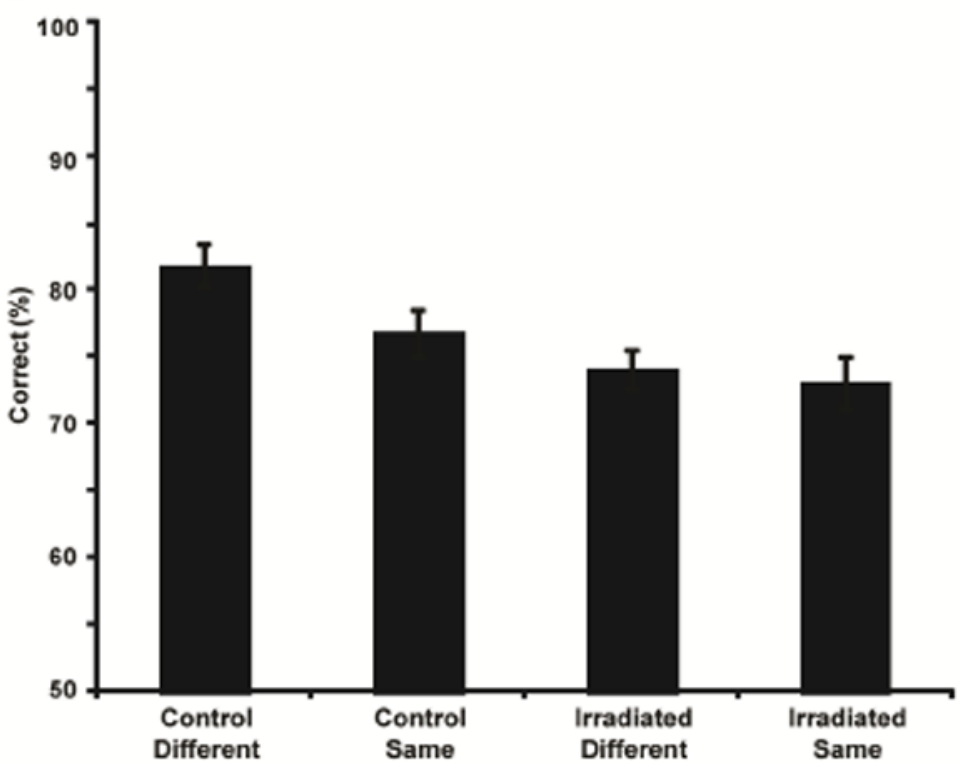

Figure 2.

Olfactory discrimination performance. In panel A, the average percent correct choices are shown for control (open symbols) and irradiated rats (filled symbols) and for the different context (solid lines) and same context conditions (dashed lines). Performance data are shown for the final session of list 1 training (Last) and the five training sessions of list 2 . In panel B, the same data are shown averaged across all five days of training on list 2. 


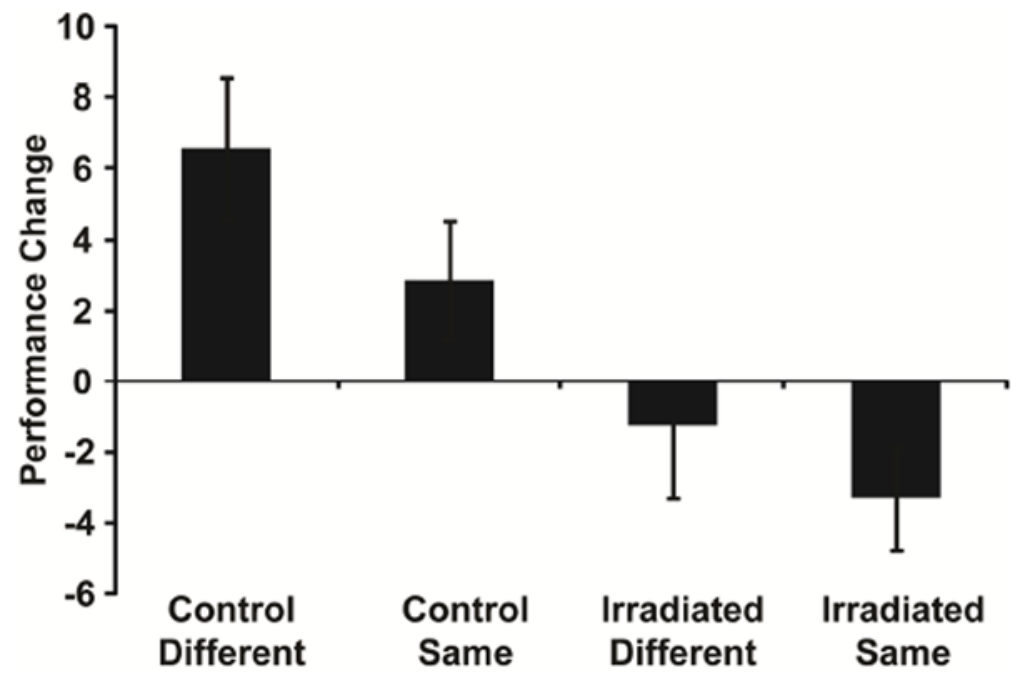

Figure 3.

Neurogenesis and Interference. Change in performance from list 1 to list 2, computed as the average percent correct during the first three sessions of list 2 minus the average percent correct during the first three sessions of list 1, is shown for each of the experimental groups. Facilitation is indicated by better performance on list 2 than on list 1 (positive values) while interference is indicated by worse performance on list 2 (negative values). 

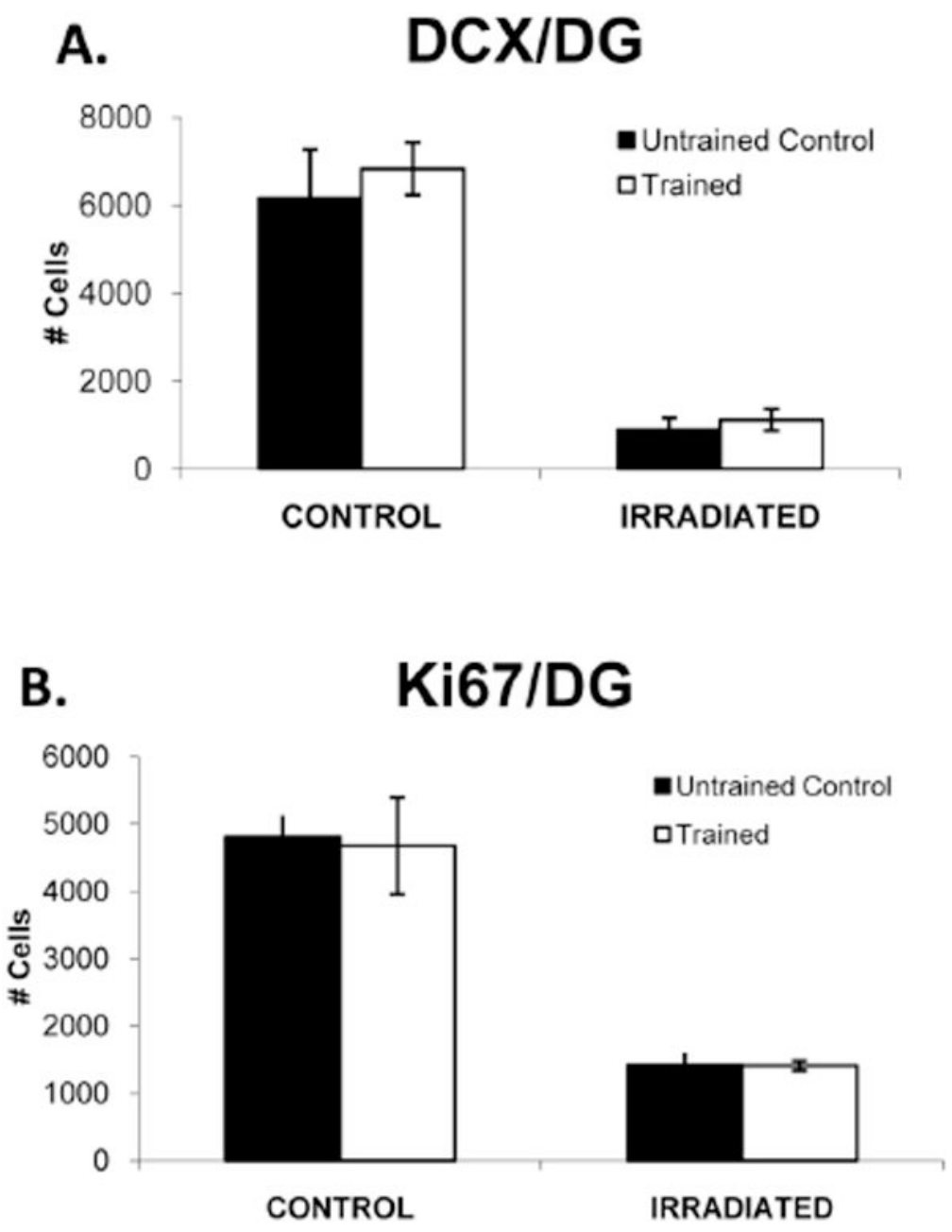

Figure 4.

Olfactory discrimination training and neurogenesis. Estimates of neurogenesis in rats trained in the olfactory discrimination task and untrained control rats are shown for irradiated and control subjects. The number of DCX+ cells per dentate gyrus is shown in A and the number of Ki67+ cells is shown in B. The trained group includes rats from the different context and same context training conditions since these groups did not differ. 

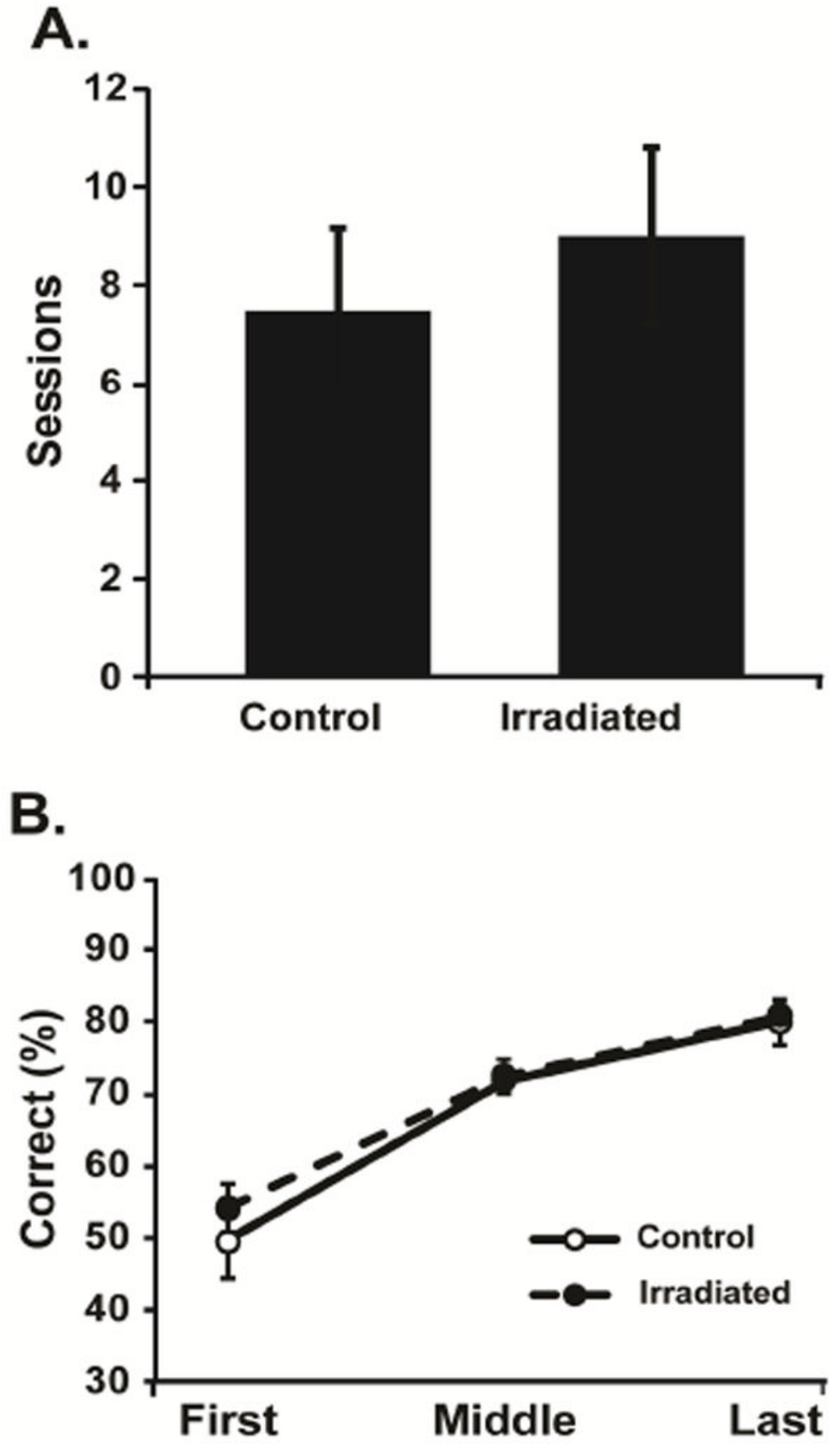

Figure 5.

Plus maze performance. The number of sessions needed to reach the behavioral criterion in the plus maze task are shown in A. Plot B illustrates the percentage of trials with a correct response for control (open) and irradiated rats (filled). Data are shown for the first training session, the session midway through training and the final training session. 


\section{A. BrdU/DG}

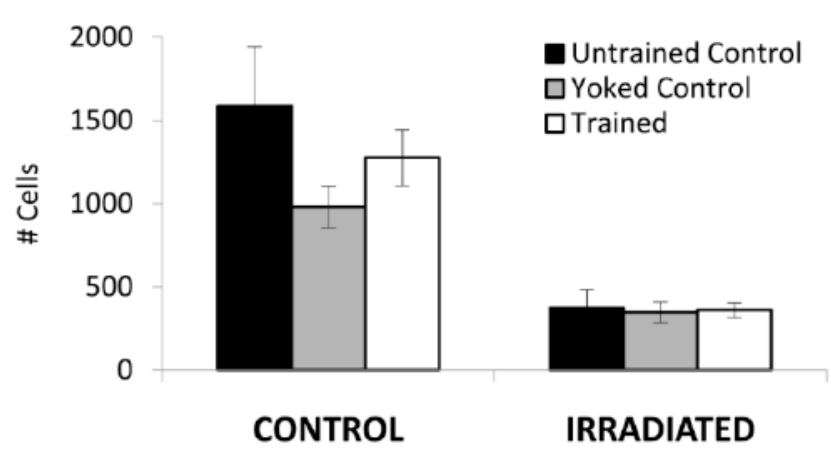

B. $\quad \%$ BrdU/CaBP

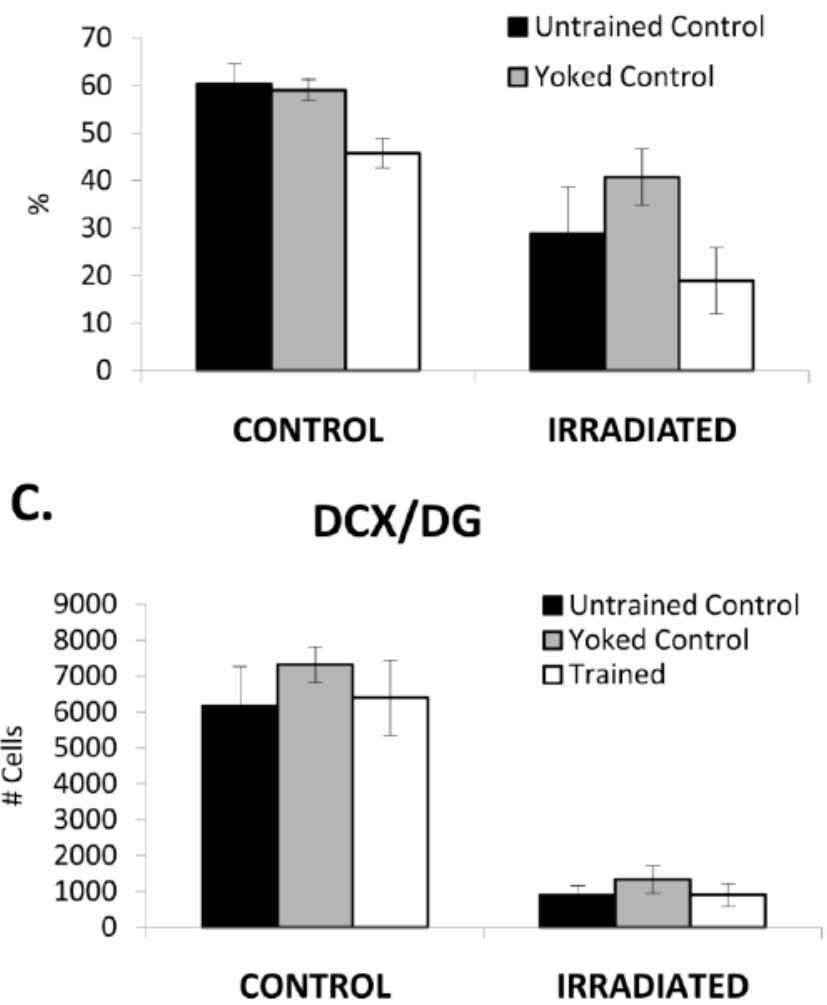

Figure 6.

Plus maze training and neurogenesis. Effects of spatial learning on neurogenesis are shown for rats trained in the plus maze task, for yoked controls (see Methods for details) and for untrained, cage controls. The survival of BrdU+ cells born 1 week before the start of training was not affected by learning (trained group) nor was it affect by handling or exposure to the training apparatus (yoked controls), compared to the untrained controls (plot A). Similarly, the rate of maturation as indicated by $\mathrm{CaBP} / \mathrm{BrdU}$ labeling was the same in trained rats, yoked controls and untrained controls (plot B). The density of DCX+ cells was also the same in trained rats, yoked controls and untrained controls (plot $\mathrm{C}$ ). 